UCRL-JC-124912

Preprint

\title{
Ultrahigh Spatial-Frequency, High-Contrast Periodic Structures Produced by Interference Lithography
}

\author{
H. T. Nguyen, J. A. Britten, R. D. Boyd, \\ B. W. Shore, M. D. Perry
}

This paper was prepared for submittal to 1996 Diffractive Optics and Micro Optics Topical Meeting

Boston, MA

April 27, 1996

April 1996

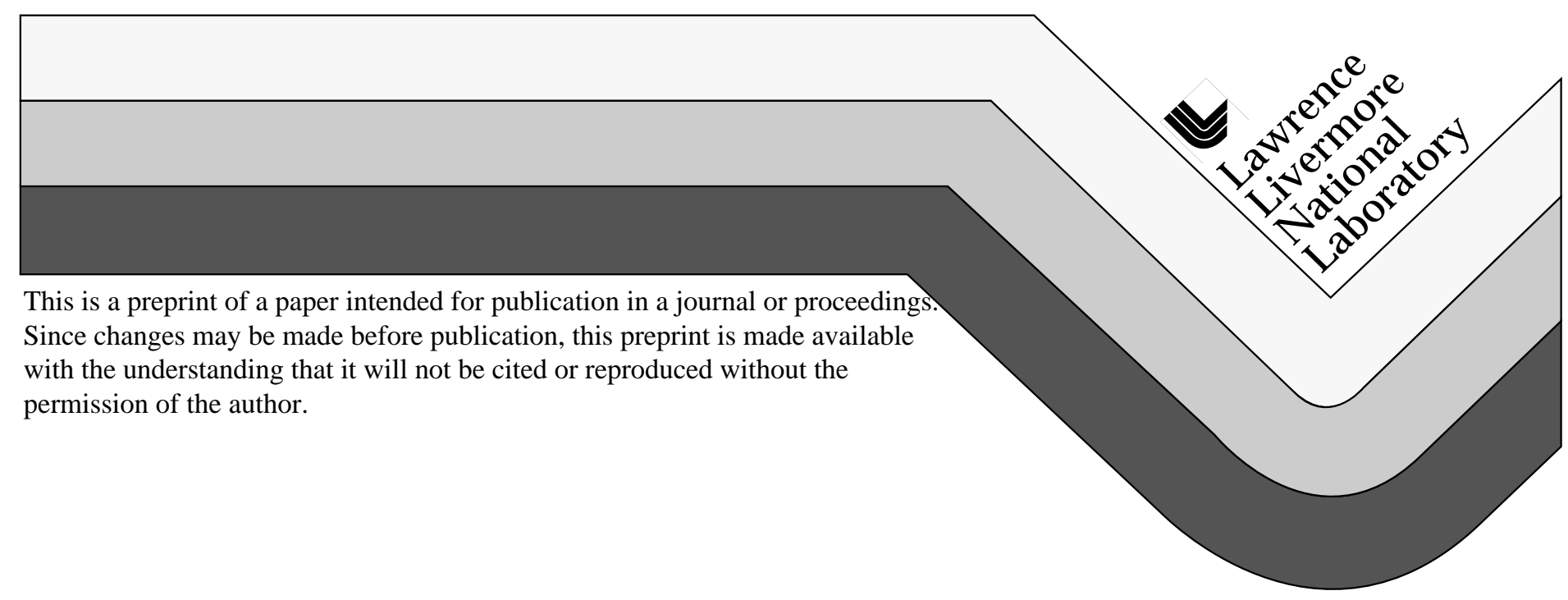




\section{DISCLAIMER}

This document was prepared as an account of work sponsored by an agency of the United States Government. Neither the United States Government nor the University of California nor any of their employees, makes any warranty, express or implied, or assumes any legal liability or responsibility for the accuracy, completeness, or usefulness of any information, apparatus, product, or process disclosed, or represents that its use would not infringe privately owned rights. Reference herein to any specific commercial product, process, or service by trade name, trademark, manufacturer, or otherwise, does not necessarily constitute or imply its endorsement, recommendation, or favoring by the United States Government or the University of California. The views and opinions of authors expressed herein do not necessarily state or reflect those of the United States Government or the University of California, and shall not be used for advertising or product endorsement purposes. 


\title{
Ultrahigh Spatial-Frequency, High-Contrast Periodic Structures Produced by Interference Lithography
}

\author{
H.T. Nguyen, J.A. Britten, R.D. Boyd, B.W. Shore, and M. D. Perry \\ Lawrence Livermore National Laboratory, P.O. Box 808, L-443, Livermore, CA 94550 \\ Tel. (510) 423-2111, FAX (510) 422-9294
}

\begin{abstract}
We have developed a process to produce high-aspect ratio, high-frequency periodic profiles recorded in a photoresist layer by interference lithography. We are able to independently control the period, duty cycle, and sidewall angle of the profiles. Highcontrast diazonapthoquinone-based photoresist and single mode operation of the exposure laser are critical. The high-aspect ratio profiles are necessary for many applications such as transfer etch masks for etching oxides and field emitter array patterning.
\end{abstract}

This work was performed under the auspices of the U. S. Department of Energy by Lawrence Livermore National Laboratory under contract No. W-7405-Eng-48. 


\title{
Ultrahigh Spatial-Frequency, High-Contrast Periodic Structures Produced by Interference Lithography
}

\author{
H.T. Nguyen, J.A. Britten, R.D. Boyd, B.W. Shore, and M. D. Perry \\ Lawrence Livermore National Laboratory, P.O. Box 808, L-443, Livermore, CA 94550 \\ Tel. (510) 423-2111, FAX (510) 422-9294
}

\section{Introduction}

During efforts to produce multilayer high efficiency dielectric reflection gratings in oxides, $351 \mathrm{~nm}$ high efficiency transmission gratings, and other development work, we required very high-contrast grating profiles in photoresist. High-contrast profiles are profiles with very steep sidewalls, greater than 80 degrees. It is quite difficult to achieve high-contrast profiles using interference lithography. The electric field distribution is sinusoidal. Therefore, one would conclude that the profile would resemble a sinusoid, as shown in Figure 1a. Early work with interference lithography produced grating profiles similar to the ones shown in Figure 1a. ${ }^{1-3}$ We have learned that if great care is taken in the processing steps, very different profiles can be achieved. Figure $1 \mathrm{~b}$ shows a very highcontrast, high-aspect ratio grating profile in photoresist. The difference between Figure 1a and Figure $1 \mathrm{~b}$ is that 1 ) the photoresist profile in Figure $1 \mathrm{~b}$ has completely developed through to the substrate, and 2) the contrast characteristics of the photoresist used in Figure $1 \mathrm{~b}$ are superior over the photoresist used in Figure 1a.
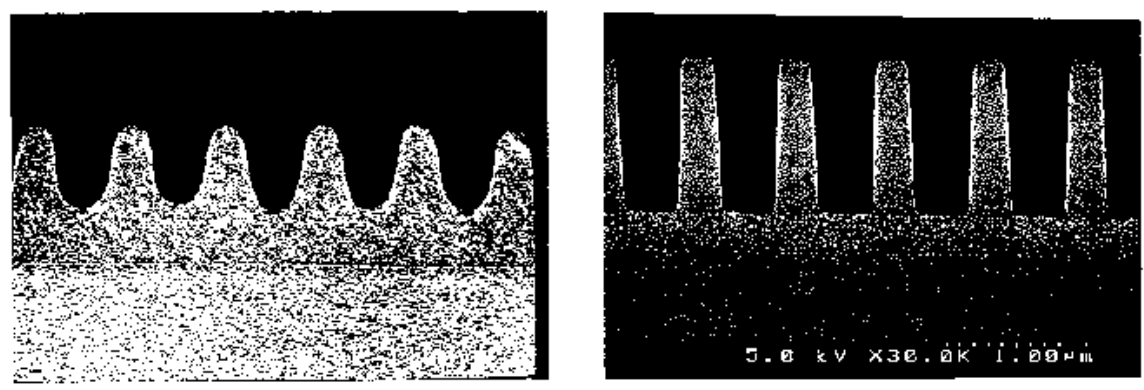

Figure 1a) Scanning electron micrograph of sinusoidal profile in photoresist, 1b) Scanning electron micrograph of high-contrast profile photoresist.

In addition to high-contrast photoresist and the complete dissolution of the grating troughs to the substrate, the exposure laser must operate single-frequency. This is essential 
for the production of high-aspect ratio, high-contrast grating profiles since the contrast of the exposure laser is transferred to the photoresist during exposure.

Etched structures such as grating profiles etched into a $\mathrm{SiO}_{2}$ layer atop a multilayer dielectric stack, and a ultraviolet transmission grating etched into bulk fused silica, require high-contrast for high-efficiency. These are more easily made with a high-contrast mask.

It is also important to mention that a high-contrast profile is not always desired or needed for all applications. In fact, a distorted sinusoidal profile is used for all of our metallic overcoated gratings. ${ }^{4}$ Additionally, we have developed a process to control the duty cycle, the full-width-at-half-maximum of the structure divided by its period, of the structures from 0.6 to 0.17 .

\section{Vertical Sidewalls}

The use of different types of photoresist has become very important to our processing technique. We have discovered that each of the different photoresists used have individual advantages and disadvantages. By choosing a particular photoresist we can now control the photoresist profiles, from a distorted sinusoid to a rectangular profile, from a duty cycle of greater than 0.6 to less than 0.17 . For instance, Photoresist B used to produce very steep side walls has excellent contrast characteristics but low sensitivity,

requiring exposure fluence $>120 \mathrm{~mJ} / \mathrm{cm}^{2}$. On the other hand, Photoresist A has very high sensitivity, requiring an exposure fluence of about $20 \mathrm{~mJ} / \mathrm{cm}^{2}$, but its contrast characteristics are lower. Figures $2 \mathrm{a}$ and $2 \mathrm{~b}$ show grating profiles of these photoresist exposed under similar conditions.
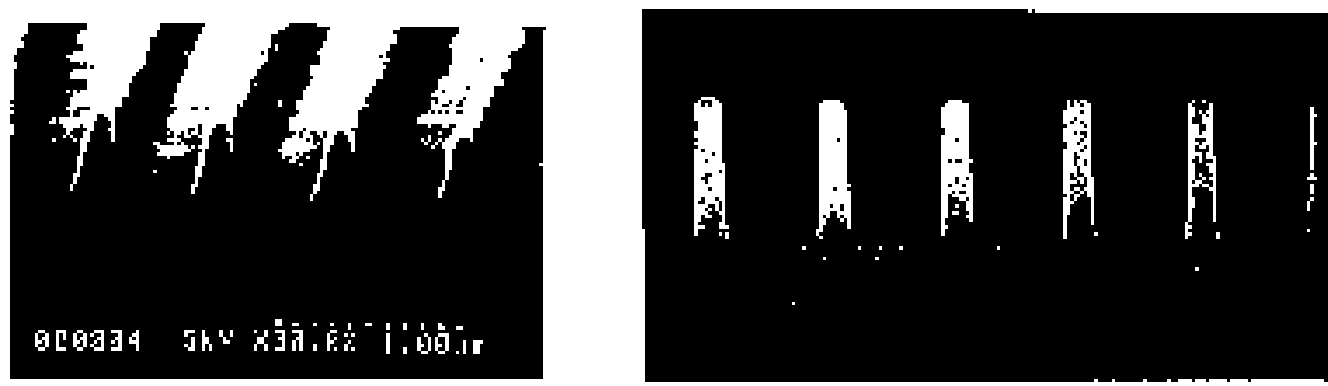

Figure 2a) Typical profiles produced with Photoresist A, 2b) Typical high-contrast profile produced with Photoresist B

The difference in the grating profiles shown is a function of the photoresist used. Even though all of the resist are diazonapthoquinone-based (DNQ), they exhibit different contrast characteristics. The basic differences between these resist are the transparency of the resin, synthesizer, and concentration of the photoactive compound. Photoresist B uses a more transparent resin which results in less UV light being absorbed, and lateral scattering yielding steeper side walls. 
The contrast differences exhibited by these two photoresist is very favorable. It allows us to control the sidewall angle of the profiles. Steep vertical side walls can be produced by using Photoresist B, while Photoresist A yields a more sinusoidal profile. We can therefore tailor the side wall angle of the photoresist profile for a specific application.

We are currently using Photoresist A for all overcoated metallic gratings. ${ }^{4}$ There is no need for steep side walls profile in this application. The distorted sinusoid metallic gratings exhibit diffraction efficiency $>90 \%$ from $800 \mathrm{~nm}$ to $1100 \mathrm{~nm}$ laser light. ${ }^{5}$ Typical profiles are shown in Figure 3. Photoresist A is a very forgiving resist because of its linear properties. If Photoresist $\mathrm{A}$ is underexposed, compensation can be made by overdeveloping. In contrast, Photoresist B has a very sharp turn-on due to its high contrast characteristic. Therefore, if it is under-exposed, over-development cannot compensate.

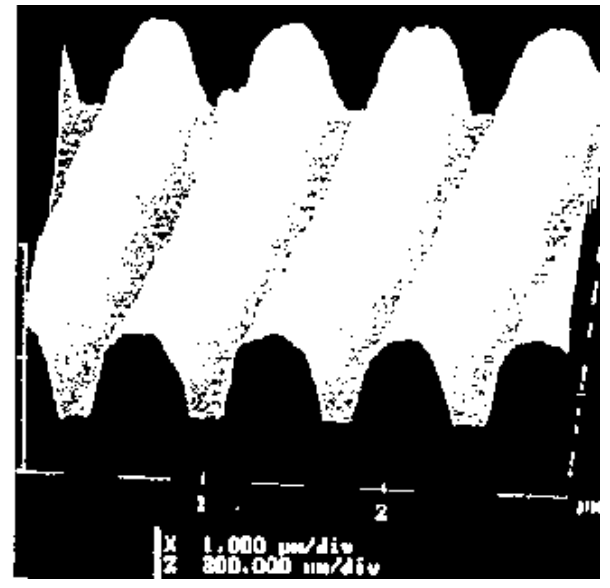

Figure 3. Typical profile for high efficiency gold-overcoated gratings.

\section{Controlling the Linewidth}

Each photoresist has a characteristic minimum linear resolution. The linewidth of a given profile can be reduce by increasing the exposure dose until the minimum linear resolution is reached for a particular photoresist, depict by the resist's LogE curve. Typically the process latitude is very small at this limit. Processing should occur where the latitude is the greatest, resulting in more controllability. Figure 4 shows a plot of the duty cycle as a function of fluence for Photoresist B. The greatest latitude occurs at a duty cycle of 0.25 with exposure latitude of $>20 \%$. The photoactive compound chemistry of the photoresist determines

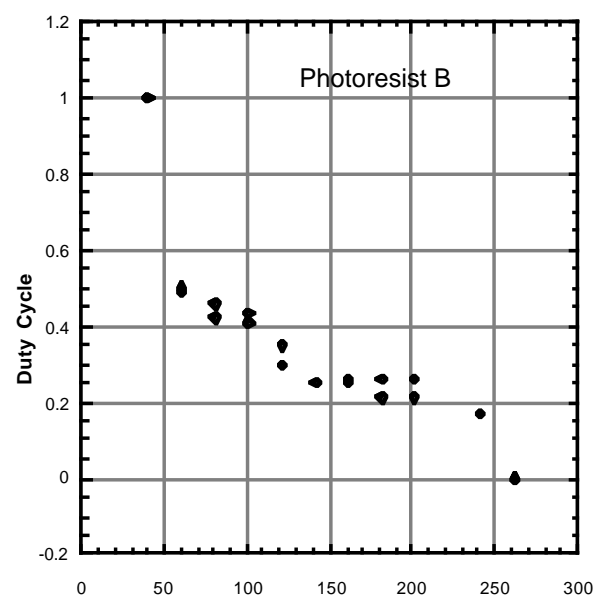

Figure 4. Plot of duty cycle as of $f$ unction of fluence. this optimum duty cycle. Different photoresist can exhibits different optimum duty cycle with similar processing latitude. Figure 5 shows photoresist profiles with different duty cycles processed under similar conditions. 

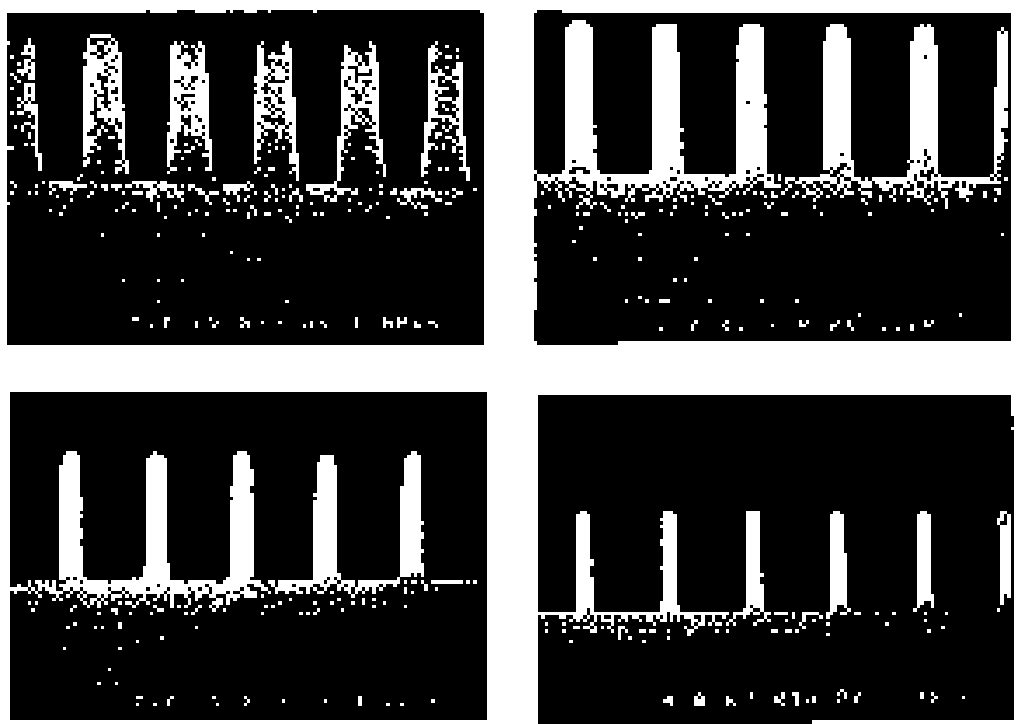

Figure 5. Micrograph of photoresist profiles with varying duty cycles produced with different photoresist.

Modeling has shown for example that high efficiency dielectric reflection grating requires a duty cycle of approximately 0.5 , while high efficiency TE gratings etched into a gold substrate requires a duty cycle of 0.2 or less. We are currently working on many different types of diffractive optics that require widely varying duty cycle and line spacing for optimum performance, and by tailoring the photoresist type used, we are able to expand the capabilities of our fabrication processes.

\section{REFERENCES}

1. R.C. Enger and S.K. Case, "High-frequency holographic transmission gratings in photoresist," J. Opt. Soc. Am. 73, 1113-1118 (1983).

2. Sten Lindau, "The groove profile formation of holographic gratings," Optica Acta 29, 13721381 (1982).

3. M.G. Moharam, T.K. Gaylord, G.T. Sincerbox, H. Werlich, and B. Yung, "Diffraction characteristics of photoresist surface-relief gratings," Appl. Opt. 23, 3214-3220 (1984).

4. R.D. Boyd, J.A. Britten, D.E. Decker, B.W. Shore, B.C. Stuart, M.D. Perry, and Lifeng Li, "High-efficiency metallic diffraction gratings for laser application," Appl. Opt. 34, 1697-1706 (1995).

5. J.A. Britten, M.D. Perry, B.W. Shore and R.D. Boyd, "A universal grating design for pulse streching and compression in 800 to $1100 \mathrm{~nm}$ range," Submitted to Optics Letters 1995. 


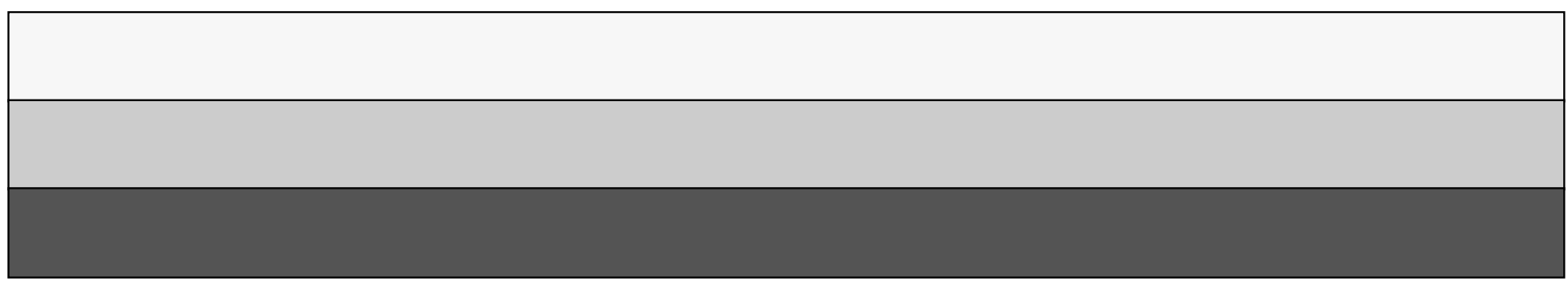

